Изв. АН Эстонии. Физ. Матем., 1989, 38, № 4, 423-432

удК 519.8

Э. ЮБН

\title{
ИСПОЛЬЗОВАНИЕ МЕТОДА НАИМЕНЬШИХ КВАДРАТОВ В МАТЕМАТИЧЕСКОМ ПРОГРАММИРОВАНИИ
}

\author{
(Представил Г. Вайникко)
}

Предлагается конечный метод нахождения решения системы недоопределенных линейных уравнений $A x=b, x \geqslant 0$, основанный на $Q R$-разложении матрицы коэффициентов. Используется высокоразвитая технология метода наименьших квадратов (МНK). Начальные значения всех переменных нулевые, $x^{0}=0$. На $k+1-\mathrm{m}$ шаге число активных переменных растет на единицу, активизируется переменная $x_{j}$, для которой минимален угол между столбцом $a_{j}$ и невязкой $b-A x^{k}$. При этом $x^{k}$ определяется $\mathrm{MHK}$ из переопределенной системы с $k$ переменными и $m$ уравнениями. Задача решена, если число активных переменных равняется числу уравнений $m$ и выполняется условие неотрицательности всех переменных (в противном случае отрицательные переменные приравниваются к нулю). Используются ортогональные преобразования Хаусхолдера и Гивенса, гарантирующие численную устойчивость алгоритма. Кроме того, выбранный критерий активизации переменных обеспечивает «максимальную» линейную независимость активных столбцов. Опыт решения задач на ЭВМ показывает эффективность предложенного метода (незначительно различаются времена решения рассматриваемой задачи и системы $A x=b)$.

\section{1. Введение}

Пусть задана $(m \times n)$-матрица $A$ и $m$ вектор $b$. Нужно найти $n$-вектор $x$, удовлетворяющий условиям

$$
\begin{array}{r}
A x=b, \\
x \geqslant 0 .
\end{array}
$$

В линейном программировании задача (1) - это нахождение начального допустимого решения. Задача квадратичного программирования сводится к определению такого решения задачи (1), которая удовлетворяет еще условиям дополняющей нежесткости.

В работе [ ${ }^{1}$ ] предлагается решить задачу (1), используя функцию Лагранжа задачи

$$
\varphi(x)=\frac{1}{2}\|A x-b\|^{2} \rightarrow \min _{x \geqslant 0}
$$

В данной работе предлагается алгоритм решения задачи (1), основанный на применении покоординатной минимизации и МНК для задачи (2). В качестве начальной точки выбирается всегда $x^{0}=0$. На каждом шаге к множеству индексов активных переменных $I J$ добавляется один элемент $j_{k+1}$. Очередная активная переменная $x_{j_{k+1}}$ определяется как решение задачи

$$
-\frac{\partial \varphi\left(x^{k}\right)}{\partial x_{j}}:\left\|a_{j}\right\|=\frac{\partial}{\partial x_{j}} \frac{1}{2}\left\|b^{k}-A^{k} x^{k}\right\|^{2}:\left\|a_{j}\right\| \rightarrow \max _{j=1, \ldots, n},
$$

где $x^{k}$ - текущая точка, вычисленная с помощью $\mathrm{MHK}, A^{k}, b^{k}-$ коэффициенты системы после $k$-го преобразования, $A^{0}=A, b^{0}=b, k=$ $=0,1 \ldots$ Если некоторый столбец $a_{j}$ есть линейная комбинация активных столбцов, то $\varphi_{x_{f}}^{\prime}\left(x^{k}\right)=0$, так как значение $\varphi(x)$ не убывает при неизменном подпространстве, натянутом на активные столбцы мат- 
рицы $A$ (см. лемму 1 в Приложении). Поэтому критерий (3) исключает активизацию почти линейно-зависимого набора столбцов $A$.

После дополнения индексного множества $I J$ новым элементом $j_{k+1}$ решается задача (2) с помощью МНК при нулевых значениях неактивных переменных. Для этого к подматрице $A$, составленной из активных столбцов и преобразованной на предыдущих шагах к треугольному виду, присоединяется очередной активный столбец. В нем преобразованием Хаусхолдера аннулируется нужное количество элементов для получения треугольной системы. Эта система может быть легко решена для проверки условия $x \geqslant 0$. При нарушении этого условия производится замена - из актива исключается столбец, соответствующий последней отрицательной переменной, оставшиеся активные столбцы снова преобразуются к треугольному виду преобразованиями Гивенса и определяется очередная активная переменная. Когда число активных переменных равняется числу уравнений $m$ и $x \geqslant 0$, задача (1) решена. Важно отметить, что определить очередную активную переменную по формуле (3) можно, и не решая треугольной системы. Ее нужно решить только для проверки неотрицательности переменных. Для ускорения работы алгоритма предусмотрена возможность решения треугольной системы не с первого шага, а начиная только с некоторого шага.

Предложенный метод соответствует методу 3 работы [1 ${ }^{1}$ (гл. 24). Он имеет сходство с обычным симплекс-методом в том смысле, что преобразуются все коэффициенты системы. Но в отличие от симплексметода преобразование производится здесь по столбцам, а не по строкам системы.

В разделе 2 приводится краткая характеристика ортогональных преобразований Хаусхолдера и Гивенса. Раздел 3 содержит подробное описание предлагаемого метода. Четвертый раздел посвящен обсуждению результатов вычислительных экспериментов. Некоторые вспомогательные утверждения доказываются в Приложении.

\section{2. Ортогональные преобразования Хаусхолдера и Гивенса}

В качестве вычислительного аппарата решения задач МНК основоположники современной теории Дж. Голуб и Ч. Лоусон выделили ортогональное преобразование Хаусхолдера. Это преобразование характеризуется численной устойчивостью, гибкостью, позволяющей легко приспосабливаться к операциям присоединения или удаления столбцов, и меньшим объемом памяти по сравнению с модифицированным методом Грама-Шмидта или методом нормальных уравнений.

Опишем коротко построение преобразования Хаусхолдера в том виде, как оно используется в настоящей работе. В задаче МНК нужно построить такое ортогональное преобразование $Q$, чтобы переопределенная система

$$
\begin{gathered}
A^{\prime} x^{\prime}=b \\
m>n^{\prime}
\end{gathered}
$$

преобразовалась к треугольному виду

$$
Q A^{\prime} x^{\prime}=Q b,
$$

где $A^{\prime}-\left(m \times n^{\prime}\right)$-матрица, $b-m$-вектор, $x^{\prime}-n^{\prime}$-вектор, $Q-(m \times m)$ матрица. На первом шаге описываемого в следующем разделе алгоритма матрица $A^{\prime}$ - это один столбец матрицы $A$ задачи (1), на втором шаге $A^{\prime}$ состоит из двух столбцов и т. д. При получении отрицательных $x_{j}$ на некоторых шагах число столбцов $A^{\prime}$ уменьшается. Для преобразования системы к треугольному виду используется на каждом 


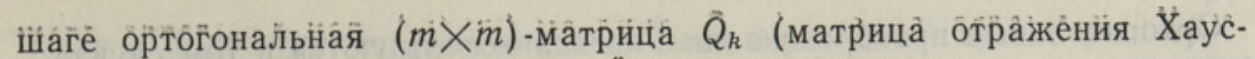
холдера), преобразующая заданный $m$-вектор $v$ к виду

$$
Q_{k} v=\left|\begin{array}{c}
v_{1} \\
\vdots \\
\dot{v}_{k-1} \\
-\sigma \sqrt{v_{k}^{2}+v_{k+1}^{2}+\cdots+v_{m}^{2}} \\
0 \\
\vdots \\
0
\end{array}\right|
$$

где $\sigma=\left\{\begin{array}{l}+1, \text { если } v_{k} \geqslant 0, \\ -1, \text { если } v_{k}<0, k=1, \ldots, m-1 .\end{array}\right.$

Геометрически нужно для этого преобразования подобрать такую гиперплоскость, чтобы координаты отражения $Q_{k} v$ удовлетворяли уравнению (6). Компоненты нормали этой гиперплоскости вычисляются по формулам (см. $\left.{ }^{1}\right]$, гл. 10)

$u_{1}=\ldots=u_{k-1}=0, \quad u_{k}=v_{h}+\sigma \sqrt{v_{k}^{2}+\ldots+v_{m}^{2}}, \quad u_{k+1}=v_{k+1}, \ldots, u_{m}=v_{m}$.

Рис. 1.
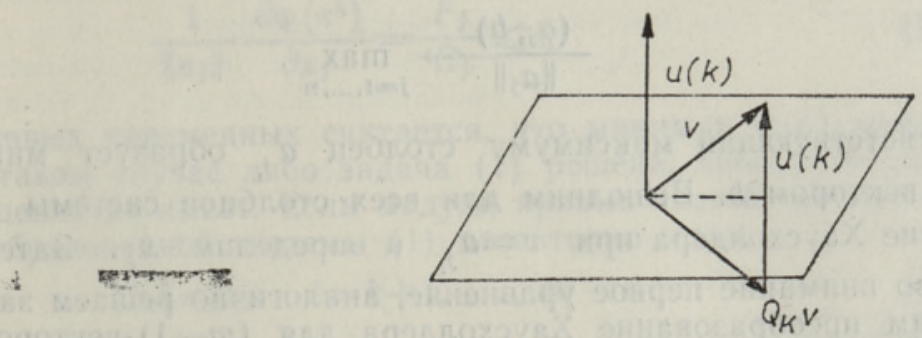

Гиперплоскости подбираются так, чтобы аннулировать в матрице $Q A^{\prime}$ системы (5) все элементы ниже главной диагонали. Матрица отражения $Q_{k}$ определяется по формулам

$$
\begin{aligned}
& Q_{k}=I_{m}+\beta_{k}^{-1} u(k) u(k)^{\mathrm{T}} \\
& \beta_{k}=-\|u(k)\|^{2} / 2,
\end{aligned}
$$

где $I_{m}-$ единичная матрица, $u(k)-$ нормаль гиперплоскости при $k$-м отражении, $\|u(k)\| \neq 0$. Ввиду простоты формулы (8) нет необходимости явно вычислять матрицу $Q_{k}$. Для нахождения произведения $\tilde{c}=Q_{k} c$, где $c-$ некоторый $m$-вектор, нужно воспользоваться формулами

$$
\begin{gathered}
t_{k}=\beta_{k}^{-1}(u(k), c), \\
\tilde{c}=c+t_{k} u(k),
\end{gathered}
$$

которые следуют из (8).

Вращения Гивенса применяются в том случае, когда нужно аннулировать только один элемент двумерного вектора $v=\left(v_{1}, v_{2}\right)^{\mathrm{T}}$. Положим

$$
c=\frac{v_{1}}{\sqrt{v_{1}^{2}+v_{2}^{2}}}, \quad s=\frac{v_{2}}{\sqrt{v_{1}^{2}+v_{2}^{2}}}, \quad G=\left(\begin{array}{rr}
c & s \\
-s & c
\end{array}\right) .
$$

Тогда $G v=\left(\begin{array}{c}\sqrt{v_{1}^{2}+v_{2}^{2}} \\ 0\end{array}\right)$. 
Оิртогональные преобразования Гивенса используются в том случа̇ѐ, когда на некотором шаге при решении системы (5) оказывается, что не выполняется условие неотрицательности, $x_{j}<0$. В таком случае из актива удаляется $j$-й столбец и для восстановления треугольного вида системы (5) нужно выполнить серию вращений Гивенса, аннулировать в каждом столбце, следующем в активе после $j$-й матрицы $Q A^{\prime}$, один элемент ниже главной диагонали. $\left[{ }^{1}\right]$.

Более подробно ортогональные преобразования рассматриваются в

\section{3. Обоснование и описание алгоритма}

Обозначим через $\left(a_{i}, a_{j}\right)$ скалярное произведение столбцов $a_{i}$ и $a_{j}$, $\left(a_{i}, a_{j}\right)=\sum_{s=1}^{m} a_{s i} a_{s j}$. Находим

$$
\frac{\partial \varphi}{\partial x_{j}}=\sum_{s=1}^{m} a_{s j}\left[\left(A_{s}, x\right)-b_{s}\right]=\sum_{s=1}^{n}\left(a_{j}, a_{s}\right) x_{s}-\left(a_{j}, b\right),
$$

где $A_{s}$ обозначает $s$-ю строку матрицы $A$.

На первом шаге при $x^{0}=0$ согласно критерию (3) активизируется та переменная, которая решает задачу

$$
\frac{\left(a_{j}, b\right)}{\left\|a_{j}\right\|} \rightarrow \max _{j=1, \ldots, n} .
$$

Соответствующий максимуму столбец $a_{j_{t}}$ образует минимальный угол с вектором $b$. Выполним для всех столбцов системы (1) преобразование Хаусхолдера при $v=a_{j_{1}}$ и определим $x_{j_{1}}$. Затем, не принимая во внимание первое уравнение, аналогично решаем задачу (12), выполним преобразование Хаусхолдера для $(m-1)$-векторов и определим $x_{j_{2}}$. Для определенности предположим, что на первом шаге активизируется $x_{1}$, на втором $-x_{2}$. Тогда

$$
\begin{array}{r}
a_{11}^{2} x_{1}+a_{12}^{2} x_{2}+a_{13}^{2} x_{3}+\ldots+a_{1 n}^{2} x_{n}=b_{1}^{2} \\
a_{22}^{2} x_{2}+a_{23}^{2} x_{3}+\ldots+a_{2 n}^{2} x_{n}=b_{2}^{2}, \\
a_{33}^{2} x_{3}+\ldots+a_{3 n}^{2} x_{n}=b_{3}^{2}, \\
a_{m 3}^{2} x_{3}+\ldots+a_{m n}^{2} x_{n}=b_{m}^{2}
\end{array}
$$

Здесь $a_{i j}{ }^{2}$ и $b_{i}{ }^{2}$ обозначают элементы $A$ и $b$ после двух преобразований Хаусхолдера. В дальнейшем для упрощения записи не будем указывать верхнего индекса.

Назовем переменные $x_{1}, x_{2}$ и соответствующие им первую и вторую строку активными, а $x_{3}=\ldots=x_{n}=0-$ пассивными. Активные переменные $x_{1}, x_{2}$ определяются из треугольной системы

$$
\begin{aligned}
a_{11} x_{1}+a_{12} x_{2} & =b_{1}, \\
a_{22} x_{2} & =b_{2} .
\end{aligned}
$$

Частные производные от функции $\varphi(x)$ в формуле (3) могут быть вычислены по системе (13), не решая треугольной системы (14). Для нахождения $\varphi_{x_{j}}^{\prime}\left(x^{2}\right), j=3, \ldots, n$, по системе (13) достаточно воспользоваться в формуле $(12)$ ( $m-2)$-векторами, так как первые два уравнения этой системы уже выполняются. 
На $k$-м шаге для определения вводимой переменной нужно решить задачу, аналогичную (12)

$$
R E=\max _{j} \frac{F_{j}}{G_{j}}
$$

где $F_{j}=\sum_{s=k}^{m} a_{s j} b_{s}, \quad G_{j}=\sqrt{a_{k j}^{2}+a_{k+1 j}^{2}+\ldots+a_{m j}^{2}}$,

и максимум находится по всем неактивным переменным, для которых $G_{j} \neq 0$. Для тех неактивных столбцов, которые являются линейной комбинацией активных $G_{j}=F_{j}=0$. Ввиду ортогональности преобразования Хаусхолдера скалярные произведения столбцов остаются неизменными, поэтому величины $F_{j}$ и $G_{j}$ могут быть вычислены рекуррентно, новые

$$
F_{j}=F_{j}-a_{k j} b_{k}, \quad G_{j}^{2}=G_{j}^{2}-a_{k j}^{2}, \quad j=1, \ldots, n .
$$

В общем случае для решения задачи может потребоваться менее $m$ шагов. Например, когда существует столбец $a_{j}, a_{j}=b$, решение получается за один шаг. Если не принимать во внимание ошибок округления, то максимальное число активных переменных не превосходит $\operatorname{rank} A$. Используются два малых положительных параметра $\varepsilon_{1}$ и $\varepsilon_{2}$. При выполнении неравенства

$$
\frac{1}{\left\|a_{j}\right\|} \frac{\partial \varphi\left(x^{k}\right)}{\partial x_{j}}=\frac{F_{j}}{G_{j}} \leqslant \varepsilon_{1}
$$

для всех пассивных переменных считается, что минимум $\varphi(x)$ найден $($ см. $[2,3])$. В таком случае либо задача (1) решена, либо установлено, что она решения не имеет. Если модули правых частей неактивных уравнений преобразованной системы (1) достаточно малы,

$$
\left|b_{i}\right| \leqslant \varepsilon_{2}, \quad i=k+1, \ldots, m,
$$

т. е. эти уравнения выполняются с точностью $\varepsilon_{2}$ и активные уравнения тоже выполняются (с машинной точностью), то считается, что задача (1) решена (шаги 5 или 20 нижеследующего алгоритма). Если при выполнении (17) нарушается хотя бы одно из неравенств (18), считается, что задача (1) решения не имеет.

Опишем теперь подробно алгоритм VRM1 решения задачи (1). Алгоритм VRM1 $\left(A, b, I J, x, F, G, m, n, \varepsilon_{1}, \varepsilon_{2}, E T\right)$ следующий.

1. Вычислить $n$-векторы $F, G$ с координатами $F_{j}=\left(a_{j}, b\right), G_{j}=$ $=\left\|a_{j}\right\|, j=1, \ldots, n$.

2. Положить число активных переменных $k:=0$.

3. Определить очередную активную переменную $x_{j_{k+1}}$ по формуле (15). Записать индекс $j_{k+1}$ в массив индексов активных переменных $I J$.

4. Если $R E<\varepsilon_{1}$, перейти к шагу 5 , в противном случае к 7 .

5. Проверить неравенство $\left|b_{i}\right| \leqslant \varepsilon_{2}$ для $i=k+1, \ldots, m$. Если все они выполняются, то перейти к шагу 21 , в противном случае к 6 .

6. Система (1) решения не имеет.

7. Увеличить число активных переменных на единицу, $k:=k+1$.

8. Проверить неравенство $k<m$. Если оно выполняется, перейти к шагу 9 , в противном случае к 12 .

9. Выполнить преобразование Хаусхолдера с вектором $v=a_{j_{k}}$ для столбцов $A, b$.

10. Вычислить новые $\tilde{F}_{j}=F_{j}-a_{k j} b_{k}, G_{j}^{2}=G_{j}^{2}-a_{k j}^{2} \cdot$

11. Проверить неравенство $k \leqslant m \times E T$. Если оно выполняется, перейти к шагу 3 , в противном случае к 12 . 
12. Решить треугольную систему типа (14) для нахожденй активных $x_{j}$.

13. Проверить для $k-1$ значений $j$ неравенство $x_{j} \geqslant 0, j \in I J$. Если все неравенства выполняются, перейти к шагу 19, в противном случае к 14 .

14. Удалить из актива индекс $j$, если $x_{j}<0$, и положить $x_{j}:=0$.

15. Привести матрицу из активных столбцов вращениями Гивенса к треугольному виду после удаления $j$-го столбца.

16. Вычислить новые $\widetilde{F}_{j}=F_{j}+a_{k j} b_{k}, G_{j}^{2}=G_{j}^{2}+a_{k j}^{2}, j=1, \ldots, n$.

17. Уменьшить число активных переменных на единицу, $k:=k-1$.

18. Перейти к шагу 3.

19. Проверить неравенство $k<m$. Если оно выполняется, перейти к шагу 20 , в противном случае к 21 .

20. Проверить неравенство $\left|b_{i}\right| \leqslant \varepsilon_{2}$ для $i=k+1, \ldots, m$. Если все они выполняются, перейти к шагу 21 , в противном случае к 3 .

21. Решение $x$ найдено.

На 11 -м шаге используется параметр $E T, 2 / m<E T \leqslant 1$, см. лемму 2 в Приложении. До величины $k=m \times E T$ условие $x \geqslant 0$ не проверяется. Опыт решения задач на ЭВМ показывает, что при $k<m / 3$ переменные $x_{j}$ почти никогда не бывают отрицательными. На 13-м шаге не проверяется условие неотрицательности последней активной переменной $x_{I J(k)}$, так как при положительном максимуме в формуле (15) для системы из неактивных уравнений $k+1, \ldots, m$ вновь выполняются условия леммы 2 Приложения и поэтому $x_{I J(k)} \geqslant 0$. На 14-м шаге в случае нескольких отрицательных переменных из актива удаляется только та переменная, которая активизировалась позже всех. В таком случае требуется минимальное число вращений на 14-м шаге. Остальные отрицательные переменные, если они имеются, могут после этого удаления стать неотрицательными.

Конечность алгоритма следует из того, что значения $\varphi(x)$ строго убывают на каждом шаге (см. лемму 3 Приложения).

\section{4. Результаты вычислений}

Пр и мер 1.

\begin{tabular}{|c|c|c|c|c|c|}
\hline & & $x_{1}$ & $\begin{array}{r}-x_{2} \\
+x_{2} \\
x_{2}\end{array}$ & $\begin{array}{l}+x_{3} \\
+x_{3}\end{array}$ & $\begin{aligned}-x_{4} & = \\
& =\end{aligned}$ \\
\hline & $\boldsymbol{F}_{j}$ & $3 / 2$ & $5 / 2$ & $3 / 2$ & $-3 / 2$ \\
\hline & $G_{j}$ & 1 & 10 & $\sqrt{2}$ & 1 \\
\hline
\end{tabular}

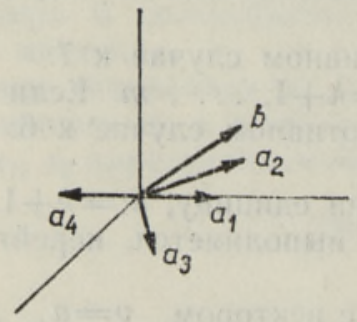

Рис. 2.

Величины $F_{j}$ и $G_{j}$ вычисляются по формуле (16). На первом шағе согласно формуле (15) активизируется $x_{1}$. Преобразуем столбцы по формулам $(6)-(9)$ при $v=(0,1,0)^{\mathrm{T}}$. 
$\begin{aligned}-x_{1}-x_{2}-x_{3}+x_{4} & =-3 / 2, \\ x_{2}-x_{3} & =0, \\ x_{2} & =1 .\end{aligned}$

Теперь $\frac{F_{j}}{G_{j}}=\frac{0}{0}, \frac{1}{\sqrt{2}}, \frac{0}{1}, \frac{0}{0}$.

Из треугольной системы определим $x_{1}=3 / 2>0$, остальные $x_{j}=0$. На втором шаге активизируется $x_{2}, v=(1,1)^{\mathrm{T}}$. Система преобразуется к виду

$$
\begin{aligned}
-x_{1}-x_{2}-x_{3}+x_{4} & =-3 / 2 \\
-\sqrt{2} x_{2}+\frac{\sqrt{2}}{2} x_{3} & =-\frac{\sqrt{2}}{2} \\
\frac{\sqrt{2}}{2} x_{3} & =\frac{\sqrt{2}}{2}
\end{aligned}
$$

причем $\frac{F_{j}}{G_{j}}=\frac{0}{0}, \quad \frac{0}{0}, \frac{1 / 2}{\sqrt{2} / 2} \frac{0}{0}$.

Из треугольной системы

$$
\begin{aligned}
-x_{1}-x_{2} & =-3 / 2, \\
-\sqrt{2} x_{2} & =-\sqrt{2} / 2
\end{aligned}
$$

определим $x_{2}=1 / 2>0, x_{1}=1>0$.

На третьем шаге активизируется $x_{3}$. При $k=m$ преобразование Хаусхолдера не выполняется. Решение треугольной системы $x_{3}=1, x_{2}=1$, $x_{1}=-1 / 2<0$ неположительное. Из актива удаляется $x_{1}$. Для преобразования системы $(21)$ к треугольному виду нужно аннулировать $a_{22}$ и $a_{33}$. Сначала выполним по формуле $(10)$ вращения Гивенса при $v=$ $=(-1,-\sqrt{ } 2)^{\mathrm{T}}$ для элементов первой и второй строки

$$
\begin{aligned}
\frac{1}{\sqrt{3}} x_{1}+\sqrt{3} x_{2}-\frac{1}{\sqrt{3}} x_{4} & =\frac{5}{2 \sqrt{3}}, \\
-\sqrt{\frac{2}{3}} x_{1}-\frac{\sqrt{6}}{2} x_{3}+\sqrt{\frac{2}{3}} x_{4} & =-\sqrt{\frac{2}{3}}, \\
\frac{\sqrt{2}}{2} x_{3} & =\frac{\sqrt{2}}{2} .
\end{aligned}
$$

Теперь аннулируем $a_{33}$, полагая $v=(-\sqrt{6} / 2, \sqrt{2} / 2)^{\mathrm{T}}$ и преобразуя вторую и третью строку

$$
\begin{aligned}
& \frac{1}{\sqrt{3}} x_{1}+\sqrt{3} x_{2} \quad-\frac{1}{\sqrt{3}} x_{4}=\frac{5}{2 \sqrt{3}} . \\
& \frac{\sqrt{2}}{2} x_{1} \quad+\sqrt{2} x_{3}-\frac{\sqrt{2}}{2} x_{4}=\frac{3 \sqrt{2}}{4} \text {, } \\
& \frac{1}{\sqrt{6}} x_{1} \quad-\frac{1}{\sqrt{6}} x_{4}=-\frac{1}{2 \sqrt{6}}, \\
& \frac{F_{j}}{G_{j}}=\frac{-1 / 12}{1 / \sqrt{6}}, \frac{0}{0}, \frac{0}{0}, \frac{1 / 12}{1 / \sqrt{6}} .
\end{aligned}
$$


Активизируется $x_{4} ;$ решение треугольной системы $x_{4}=1 / 2, \quad x_{3}=1$, $x_{2}=1$ неотрицательно. Задача решена.

П р и м е р 2. Этот пример имеет единственное неотрицательное решение $x=(7,0,8,0,0,47,0)^{\mathrm{T}}$.

$$
\begin{aligned}
4 x_{1}+5 x_{2}+9 x_{3}+11 x_{4} & =100 \\
x_{1}+x_{2}+x_{3}+x_{4}+x_{5} & =15 \\
7 x_{1}+5 x_{2}+3 x_{3}+2 x_{4}+x_{6} & =120 \\
3 x_{1}+5 x_{2}+10 x_{3}+15 x_{4}+x_{7} & =101 .
\end{aligned}
$$

При $\varepsilon_{1}=10^{-20}, \varepsilon_{2}=10^{-16}$ было получено решение $x_{1}=6,999999999$ $99995, \quad x_{2}=0,000000000000098, \quad x_{3}=7,99999999999997, \quad x_{6}=46,999$ 999999999 97. Значение целевой функции (2) $\varphi(x)=0,13 \cdot 10^{-28}$.

Задачи были решены на ЭВM ЕC-1046 (операционная система VM) на языке ФОРТРАН-77. Использовалась двойная точность.

Наиболее трудоемки выполнение преобразования Хаусхолдера для массивов $A$ и $b$ (9-й шаг), особенно при малом $k$, и выполнение вращений Гивенса (15-й шаг) при удалении отрицательных переменных. Однако такие переменные в решенных примерах встречались редко.

Для задания элементов $A$ и $b$ использовалась стандартная подпрограмма GAUSS, генерирующая нормально распределенные случайные величины при заданной средней $A M$ и дисперсии $\sigma^{2}$. Через $K L$ обозначается суммарное количество шагов, связанных с добавлением или удалением переменных.

\begin{tabular}{lrrcccccccc} 
Номер & $m$ & $n$ & $E T$ & $\varepsilon_{1}$ & $\varepsilon_{2}$ & $A M$ & $\sigma$ & $K L$ & $\varphi(x)$ & Время \\
\hline 3. & 40 & 120 & 0,33 & $10^{-20}$ & $10^{-14}$ & 0 & 10 & 42 & $0,2 \cdot 10^{-26}$ & $12^{\prime \prime}$ \\
4. & 40 & 60 & 0,33 & $10^{-20}$ & $10^{-14}$ & 0 & 10 & 21 & $0,1 \cdot 10^{4}$ & $8^{\prime \prime}$ \\
5. & 70 & 450 & 0,25 & $10^{-25}$ & $10^{-14}$ & 10 & 100 & 78 & $0,1 \cdot 10^{-23}$ & $1^{\prime} 07^{\prime \prime}$ \\
6. & 100 & 450 & 0,33 & $10^{-25}$ & $10^{-14}$ & 1 & 10 & 102 & $0,1 \cdot 10^{-21}$ & $2^{\prime} 17^{\prime \prime}$ \\
7. & 100 & 110 & 0,25 & $10^{-20}$ & $10^{-14}$ & 1 & 10 & 46 & $0,2 \cdot 10^{4}$ & $18^{\prime \prime}$ \\
8. & 100 & 440 & 0,33 & $10^{-20}$ & $10^{-14}$ & 1 & 10 & 152 & $0,3 \cdot 10^{-24}$ & $3^{\prime} 15^{\prime \prime}$
\end{tabular}

\section{Приложение}

Для задачи $A x=b$ составим систему нормальных уравнений $\left[{ }^{4}\right]$, предпологая, что активными являются переменные $x_{1}, \ldots, x_{k}$, пассивными - остальные $x_{k+1}=\ldots=x_{n}=0$,

$$
\begin{aligned}
& \left(a_{1}, a_{1}\right) x_{1}+\ldots+\left(a_{1}, a_{k}\right) x_{k}=\left(a_{1}, b\right), \\
& \left(a_{k}, a_{1}\right) x_{1}+\ldots+\left(a_{k}, a_{k}\right) x_{k}=\left(a_{k}, b\right) .
\end{aligned}
$$

Л ем а 1. Если пассивный столбец $a_{j}$ есть линейная комбинация активных столбцов, то $\varphi_{x_{j}}^{\prime}\left(x^{k}\right)=0$.

Док аз а тель с тво. Пусть $a_{j}=\alpha_{1} a_{1}+\ldots+\alpha_{k} a_{k}$. Тогда по формуле (11)

$$
\begin{gathered}
\frac{\partial \varphi}{\partial x_{j}}=\sum_{s=1}^{n}\left(a_{j}, a_{s}\right) x_{s}-\left(a_{j}, b\right)=\sum_{i=1}^{k} \alpha_{i}\left(a_{1}, a_{i}\right) x_{1}+\ldots+\sum_{i=1}^{k} \alpha_{i}\left(a_{k}, a_{i}\right) x_{k}- \\
-\sum_{i=1}^{k} \alpha_{i}\left(a_{i}, b\right)=\sum_{i=1}^{k} \alpha_{i}\left[\sum_{s=1}^{k}\left(a_{i}, a_{s}\right) x_{s}-\left(a_{i}, b\right)\right]=0,
\end{gathered}
$$

т. к. выражения в квадратных скобках равны нулю по формуле (22).

Л ем а 2. Если задача (1) имеет решение, то на двух первых шагах метода VRM1 значения переменных $x_{j}$ неотрицательны. 
Доказательство. На первом шаге активизируется переменная $x_{j_{1}}$, решающая задачу $(12), x_{j_{1}}=\left(a_{j_{1}}, b\right) /\left\|a_{j}\right\|^{2}$ находится из системы (22). Если $\left(a_{j_{1}}, b\right)<0$, то для всех $j\left(a_{j}, b\right)<0$ и согласно формуле (11) $\varphi^{\prime} x_{j}(x=0)=-\left(a_{j}, b\right)>0$, т. е. минимум в задаче (2) единственный и достигается при $x=0$. Но тогда задача (1) не имеет решения, что противоречит предположению.

Пусть на втором шаге активизируется $x_{j_{2}}$, тогда

$$
\begin{gathered}
\frac{\partial \varphi\left(x_{j_{1}}=\left(a_{j_{1}}, b\right) /\left\|a_{j_{1}}\right\|^{2}\right)}{\partial x_{j_{2}}}=\left(a_{j_{2}}, a_{j_{1}}\right) x_{j_{1}}-\left(a_{j_{2}}, b\right)= \\
=\frac{\left(a_{j_{2}}, a_{j_{1}}\right)\left(a_{j_{1}}, b\right)}{\left\|a_{j_{1}}\right\|^{2}}-\left(a_{j_{2}}, b\right)<0 .
\end{gathered}
$$

Переменные $x_{j_{1}}$ и $x_{j_{2}}$ выразим по формуле Крамера из системы

$$
\begin{gathered}
-\frac{\left|\begin{array}{c}
\left(a_{j_{1}}, b\right)\left(a_{j_{1}}, a_{j_{2}}\right) \\
\left(a_{j_{2}}, b\right)\left\|a_{j_{2}}\right\|^{2}
\end{array}\right|}{\Delta}, \quad x_{j_{2}}=\frac{\left|\begin{array}{c}
\left\|a_{j_{1}}\right\|^{2}\left(a_{j_{1}}, b\right) \\
\left(a_{j_{1}}, a_{j_{2}}\right)\left(a_{j_{2}}, b\right)
\end{array}\right|}{\Delta}, \\
\Delta=\left|\begin{array}{c}
\left\|a_{j_{1}}\right\|^{2}\left(a_{j_{1}}, a_{j_{2}}\right) \\
\left(a_{j_{1}}, a_{j_{2}}\right)\left\|a_{j_{2}}\right\|^{2}
\end{array}\right| .
\end{gathered}
$$

По лемме 1 столбцы $a_{j_{1}}$ и $a_{j_{2}}$ линейно-независимы и $\Delta>0$ как определитель Грама. По формуле

$$
\frac{\left(a_{j_{2}}, b\right)}{\left\|a_{j_{2}}\right\|} \leqslant \frac{\left(a_{j_{1}}, b\right)}{\left\|a_{j_{1}}\right\|} \text { или }
$$

$\left(a_{j_{2}}, b\right)\left\|a_{j_{1}}\right\|\left\|a_{j_{2}}\right\| \leqslant\left(a_{j_{1}}, b\right)\left\|a_{j_{2}}\right\|^{2}$, откуда по формуле (24) следует, что $\Delta x_{j_{1}}=\left(a_{j_{1}}, b\right)\left\|a_{j_{2}}\right\|^{2}-\left(a_{j_{2}}, b\right)\left(a_{j_{1}}, a_{j_{2}}\right) \geqslant 0$. Неотрицательность $x_{j_{2}}$ следует из формулы (23).

Л ем м а 3. Метод VRM1 решает задачу (1) за конечное число шагов.

Д оказ ательство. На каждом шаге мы минимизируем $\varphi(x)$ в некотором подпространстве пространства $R^{n}$. Общее число таких подпространств конечное. Конечность метода не имеет места только в том случае, когда существует цикл - повторное появление некоторого набора активных переменных после нескольких шагов. Отсутствие цикла следует из того, что при каждом изменении множества активных переменных значения $\varphi(x)$ убывают. При добавлении новой переменной в случае удачного шага, когда $x^{k+1} \geqslant 0$, убывание $\varphi(x)$ очевидно.

Теперь рассмотрим случай, когда очередная точка $x^{k+1}$ получается из $x^{h}$ в результате замены (добавления и удаления) одной переменной. Обозначим через $y^{k+1}$ точку, получаемую из $x^{k}$ добавлением некоторой переменной, и пусть $j$-я переменная отрицательна, $y_{j}^{k+1}<0$. Еще обозначим через $z^{k+1}$ точку пересечения прямой через $x^{k}$ и $y^{k+1} \mathrm{c}$ $j$-й координатной плоскостью. Из выпуклости $\varphi(x)$ следует, что $\varphi\left(x^{k}\right)>\varphi\left(z^{k+1}\right)$. Кроме того, $\varphi\left(z^{k+1}\right) \geqslant \varphi\left(x^{k+1}\right)$, где $x^{k+1}$ обозначает точку минимума $\varphi(x)$ на следующем шаге (после исключения $j$-й и активизации $j_{k+1}$-й переменной). Следовательно, $\varphi(x)$ убывает и в случае каждой замены переменной.

В заключение хочу выразить благодарность Л. Кивистику, указавшему на ряд неточностей в первоначальном варианте статьи. 

1. Лоусон Ч., Хенсон $P$. Численное решение задач метода нанменьших квадратов.
М., Наука, 1986 .

2. Поляк Б. Т. Введение в оптимизацию. М., Наука, 1983.

3. Пиеничный Б. Н., Данилин Ю. М. Численные методы в экстремальных задачах. М., Наука, 1975.

4. Стренг Г. Линейная алгебра и ее применения. М., Мир, 1980.

Таллиннский политехнический институт

Поступила в редакцию

$18 / \mathrm{I} \quad 1989$

Переработанный вариант

$8 /$ VI 1989

\section{E. $O B I$}

\section{VÄHIMRUUTUDE MEETODI KASUTAMINE MATEMAATILISES PLANEERIMISES} meetod.

On esitatud alamääratud lineaarse võrrandisüsteemi mittenegatiivse lahendi leidmise E. $O B I$

\section{LEAST SQUARES METHOD IN MATHEMATICAL PROGRAMMING}

In this paper a finite method the objective of which is to find a non-negative solution for a linear system of ill-determined linear equations is proposed. The method is based on $Q R$-decomposition of the coefficient matrix and the least-squares approach. Initial data are supposed to take zero, $x^{0}=0$. At the $k+1$ th step of the algorithm one more variable $x_{j}$ becomes active and it is chosen so that the angle between the corresponding column $a_{j}$ and discrepancy $b-A x^{k}$ would be the smallest. The current $x^{k}$ is defined as a least squares solution for a over-determined linear system of $k$ variables and $m$ equations. The problem is considered to be solved when the number of active variables gets equal to the number of equations and all variables are non-negative (if some variable occurs to be negative it is substituted by 0 ). Orthogonal transformations by Householder and Givens are used to guarantee the numerical stability of the algorithm. Furthermore, the criterion for activizing the variables ensures «maximal» linear independence of the active columns. Numerical examples are presented. 